\title{
Aplicação de técnicas de análise numérica a um modelo de escoamento de água em áreas amazônicas alagáveis
}

\author{
Thiago F. Leal \\ PPGEM - UERJ, 20550-900, Rio de Janeiro, RJ \\ IFRJ, 26600-000, Paracambi, RJ \\ E-mail: thiagofranco@ime.uerj.br
}

\section{Cassia I. G. Silva}

IC - UFF, 24210-240, Niterói, RJ

IFRJ, 26600-000, Paracambi, RJ

E-mail: cassiaisac@ic.uff.br

\author{
Denise B. Duczmal \\ ICEX - Universidade Federal de Minas Gerais \\ 31270-010, Belo Horizonte, MG \\ E-mail: burgarelli@ufmg.br \\ Carlos A. de Moura \\ IME - Universidade do Estado do Rio de Janeiro \\ 20550-900, Rio de Janeiro, RJ \\ E-mail: demoura@ime.uerj.br
}

Resumo: O ecossistema amazônico sofre sazonalmente com alterações em seu ciclo hidrológico, ocasionando o alagamento de vastos trechos de floresta. Esse processo, entretanto, é fundamental para a manutenção de ciclos naturais. Compreender a dinâmica dessas áreas, portanto, possibilita antecipar o efeito de ações não sustentáveis. Sob tal motivação, este trabalho estuda um modelo de escoamento em áreas amazônicas alagáveis, fundamentado com as equações de Navier-Stokes. Para a obtenção do problema discreto é utilizado o Método dos Volumes Finitos. Emprega-se o Método Marker and Cell para solução das equações de Navier-Stokes. Além disso, a estrutura de dados Autonomous Leaves Graph, que traz recursos adaptativos em sua formulação, é a ferramenta empregada para simular o domínio do problema. Com isso, propõe-se observar, em experimentos preliminares, as direções do campo de velocidades da água que flui entre os troncos da floresta inundada, abrindo espaço para a avaliação de outras características do escoamento.

\section{Introdução}

A Amazônia é a maior floresta tropical do mundo, cujo território extravasa as fronteiras políticas do Brasil, estendendo-se da Cordilheira dos Andes até o Oceano Atlântico. Apresenta rica biodiversidade e todas as relações no ecossistema procedem com total harmonia.

O índice pluviométrico na região, elevado na maior parte do ano, é influenciado por fenômenos como o vapor d'água proveniente do Oceano Atlântico em direção à Cordilheira dos Andes, o processo de evapotranspiração, que é o retorno à atmosfera da umidade não aproveitada pela vegetação, além do próprio degelo decorrente do verão andino. Somados, esses fatores geram ciclos de precipitação sobre a floresta, alimentando as nascentes e os afluentes dos principais rios da bacia amazônica.

O aumento do volume de água e a oscilação do regime de chuvas fazem com que os rios transbordem e inundem grandes extensões da floresta. A amplitude de variação do nível da água dos rios pode chegar a 16 metros em algumas áreas. Ecologistas da Amazônia denominam essas variações previsíveis como pulso de inundação.

A água que circula nos trechos de floresta alagada transporta sedimentos e material orgânico para outros locais, contribuindo para a redistribuição de nutrientes e energia. Isto constitui um processo fundamental para a floresta, nutrindo a vegetação e disseminando sementes, o que favorece decisivamente a manutenção da cobertura vegetal.

Além dos benefícios ecológicos, os alagamentos anuais têm importante papel sócioeconômico. A população ribeirinha, em sua quase totalidade, sobrevive dos processos de extrativismo e exploração da biomassa animal, sobretudo de peixes e crustáceos, que ali se reproduzem e desenvolvem. Além disso, no período de retorno da água ao nível normal, a matéria orgânica diluída na água, proveniente da parte mais elevada na mata, se deposita no solo propiciando a agricultura de subsistência. 


\section{Modelo de escoamento}

Neste trabalho, temos o interesse em examinar técnicas a serem empregadas em um modelo de escoamento, baseado nas equações de Navier-Stokes de momentum e da continuidade. A formulação bidimensional do problema pode ser descrita em notação vetorial como segue, sendo (1) a equação do momentum e (2) a equação da continuidade:

$$
\begin{gathered}
\rho\left(\frac{\partial \vec{U}}{\partial t}+(\vec{U} \cdot \nabla) \vec{U}\right)=-\nabla p+\mu \nabla^{2} \vec{U}+F \\
\frac{\partial \rho}{\partial t}+\nabla \cdot(\rho \vec{U})=F_{m}
\end{gathered}
$$

onde $\vec{U}$ representa o vetor velocidade e suas componentes expressam a informação referente a cada eixo coordenado, $\rho$ a densidade do fluido, $\mu$ a viscosidade, $p$ a pressão, $F$ a fonte de movimento e $F_{m}$ fonte de massa . Os operadores exibidos estão em sua forma usual.

Assim, em coordenadas cartesianas e seguindo as considerações descritas em [4]:

$$
\begin{gathered}
u \frac{\partial u}{\partial x}+v \frac{\partial u}{\partial y}-\eta\left(\frac{\partial^{2} u}{\partial x^{2}}+\frac{\partial^{2} u}{\partial y^{2}}\right)+\frac{\partial p}{\partial x}=0 \\
u \frac{\partial v}{\partial x}+v \frac{\partial v}{\partial y}-\eta\left(\frac{\partial^{2} v}{\partial x^{2}}+\frac{\partial^{2} v}{\partial y^{2}}\right)+\frac{\partial p}{\partial y}=0 \\
\frac{\partial u}{\partial x}+\frac{\partial v}{\partial y}=0
\end{gathered}
$$

Não serão consideradas forças externas atuando sobre o escoamento, o que justifica a ausência dos termos fonte nas equações $(1(\mathrm{a}-\mathrm{b}))$ e (3). O fator $\eta=\mu / \rho$ representa a viscosidade cinemática.

Considerando, com boa aproximação, o escoamento como incompressível, é possível reescrever os termos convectivos das equações $(1(\mathrm{a}-\mathrm{b}))$ fazendo:

$$
\begin{aligned}
& u u_{x}+v u_{y} \Rightarrow\left(u^{2}\right)_{x}+(u v)_{y} \\
& u v_{x}+v v_{y} \Rightarrow(u v)_{x}+\left(v^{2}\right)_{y}
\end{aligned}
$$

As equações (1(a-b)), então, podem ser escritas como:

$$
\begin{aligned}
& \frac{\partial u}{\partial t}+\frac{\partial\left(u^{2}\right)}{\partial x}+\frac{\partial(u v)}{\partial y}-\eta\left(\frac{\partial^{2} u}{\partial x^{2}}+\frac{\partial^{2} u}{\partial y^{2}}\right)+\frac{\partial p}{\partial x}=0 \\
& \frac{\partial v}{\partial t}+\frac{\partial(u v)}{\partial x}+\frac{\partial\left(v^{2}\right)}{\partial y}-\eta\left(\frac{\partial^{2} v}{\partial x^{2}}+\frac{\partial^{2} v}{\partial y^{2}}\right)+\frac{\partial p}{\partial y}=0
\end{aligned}
$$

e será esta a notação adotada a partir deste ponto. 


\section{Abordagem numérica}

Para o tratamento numérico das equações, foi utilizado o Método MAC (Marker and Cell), proposto por [3], que é um esquema explícito para a solução das equações de Navier-Stokes. É normalmente implementado sobre uma malha deslocada (em inglês, staggered grid), onde as variáveis do escoamento não são armazenadas na mesma posição. A saber, em cada célula de centro $(i, j)$, são armazenadas:

i. A pressão $p$ em seu centro;

ii. A componente $u$ da velocidade nas faces laterais;

iii. A componente $v$ da velocidade nas faces superior e inferior.

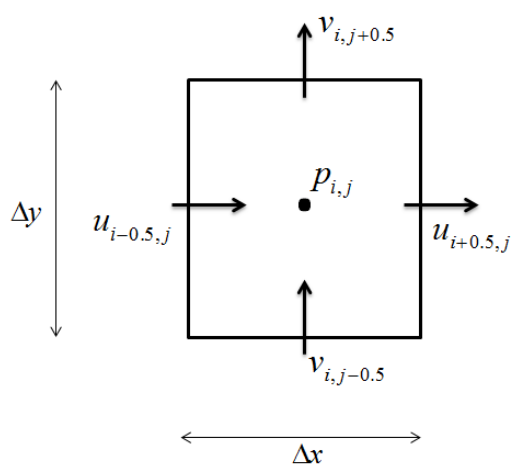

Figura 1: Célula computacional em uma malha deslocada.

Os termos envolvendo velocidades são discretizados no passo $n$, enquanto aquele que envolve a pressão é discretizado no passo $n+1$. Após o cálculo de $u^{n+1}$ e $v^{n+1}$, todas as variáveis do escoamento terão avançado no tempo.

A equação do momentum, na direção $x$, tem sua forma discreta obtida sobre o ponto $(i+0.5, j)$, utilizando as seguintes aproximações:

$$
\begin{array}{llr}
\left.\frac{\partial u}{\partial t}\right|_{i+0.5, j}=\frac{u_{i+0.5, j}^{n+1}-u_{i+0.5}^{n}}{\Delta t} & \left.\frac{\partial p}{\partial x}\right|_{i+0.5, j}=\frac{p_{i+1, j}-p_{i, j}}{\Delta x} \\
\left.\frac{\partial u^{2}}{\partial x}\right|_{i+0.5, j}=\frac{\left(u^{2}\right)_{i+1, j}-\left(u^{2}\right)_{i, j}}{\Delta x} & \left.\frac{\partial(u v)}{\partial y}\right|_{i+0.5, j}=\frac{(u v)_{i+0.5, j+0.5}-(u v)_{i+0.5, j-0.5}}{\Delta y} \\
\left.\frac{\partial^{2} u}{\partial x^{2}}\right|_{i+0.5, j}=\frac{u_{i-0.5, j}-2 u_{i+0.5, j}+u_{i+1.5, j}}{(\Delta x)^{2}} & \left.\frac{\partial^{2} u}{\partial y^{2}}\right|_{i+0.5, j}=\frac{u_{i+0.5, j+1}-2 u_{i+0.5, j}+u_{i+0.5, j-1}}{(\Delta y)^{2}}
\end{array}
$$

Assim, substituindo essas expressões em (4(a)) e efetuando simplificações, temos:

$$
u_{i+0.5, j}^{n+1}=F_{i+0.5, j}^{n}-\frac{\Delta t}{\Delta x}\left(p_{i+1, j}^{n+1}-p_{i, j}^{n+1}\right)
$$

onde

$$
\begin{aligned}
F_{i+0.5, j}^{n}= & u_{i+0.5, j}^{n}+\Delta t\left[-\frac{u^{2}{ }_{i+1, j}-u_{i, j}^{2}}{\Delta x}-\frac{(u v)_{i+0.5, j+0.5}-(u v)_{i+0.5, j-0.5}}{\Delta y}\right]+ \\
+\Delta t & {\left[\eta\left(\frac{u_{i-0.5, j}-2 u_{i+0.5, j}+u_{i+1.5, j}}{(\Delta x)^{2}}+\frac{u_{i+0.5, j+1}-2 u_{i+0.5, j}+u_{i+0.5, j-1}}{(\Delta y)^{2}}\right)\right] }
\end{aligned}
$$


é um termo que depende apenas da velocidade, na direção $x$, no passo de passo $n$.

Seguindo este padrão, é possível adaptar o processo à direção $y$, desta vez sobre $(i, j+0.5)$, obtendo:

$$
v_{i, j+0.5}^{n+1}=G_{i, j+0.5}^{n}-\frac{\Delta t}{\Delta y}\left(p_{i, j+1}^{n+1}-p_{i, j}^{n+1}\right)
$$

sendo $G_{i, j+0.5}^{n}$ um termo que depende apenas da velocidade no passo $n$, a saber,

$$
\begin{aligned}
G_{i, j+0.5}^{n}=v_{i+0.5, j}^{n}+\Delta t\left[-\frac{\left(v^{2}\right)_{i, j+1}-\left(v^{2}\right)_{i, j}}{\Delta y}-\frac{(u v)_{i+0.5, j+0.5}-(u v)_{i-0.5, j+0.5}}{\Delta x}\right]+ \\
+\Delta t\left[\eta\left(\frac{v_{i, j-0.5}-2 v_{i, j+0.5}+v_{i, j+1.5}}{(\Delta y)^{2}}+\frac{v_{i-1, j+0.5}-2 v_{i, j+0.5}+v_{i+1, j+0.5}}{(\Delta x)^{2}}\right)\right]
\end{aligned}
$$

Nesta etapa da abordagem, as velocidades ainda não respeitam o princípio da conservação de massa modelado pela equação da continuidade. A solução numérica das equações de NavierStokes passa, portanto, pela imposição da restrição global $\nabla U=0$ a cada passo de tempo. Para tal, o método envolve o cálculo da pressão em cada célula $(i, j)$. A forma discreta da equação da continuidade, no nível de passo $n+1$, é escrita como segue:

$$
\left.\frac{\partial u}{\partial x}\right|_{i, j} ^{n+1}+\left.\frac{\partial v}{\partial y}\right|_{i, j} ^{n+1}=\frac{u_{i+0.5, j}^{n+1}-u_{i-0.5, j}^{n+1}}{\Delta x}+\frac{v_{i, j+0.5}^{n+1}-v_{i, j-0.5}^{n+1}}{\Delta y}=0
$$

Através da substituição de (5) e (6) na expressão acima, tem-se:

$$
\frac{p_{i+1, j}^{n+1}-2 p_{i, j}^{n+1}+p_{i-1, j}^{n+1}}{(\Delta x)^{2}}+\frac{p_{i, j+1}^{n+1}-2 p_{i, j}^{n+1}+p_{i, j-1}^{n+1}}{(\Delta y)^{2}}=\frac{1}{\Delta t}\left(\frac{F_{i+0.5, j}^{n}-F_{i-0.5, j}^{n}}{\Delta x}+\frac{G_{i, j+0.5}^{n}-G_{i, j-0.5}^{n}}{\Delta y}\right)
$$

Esta expressão é a forma discreta de uma Equação de Poisson em $p$, no instante $n+1$, onde:

$$
b=b(i, j)=\frac{1}{\Delta t}\left(\frac{F_{i+0.5, j}^{n}-F_{i-0.5, j}^{n}}{\Delta x}+\frac{G_{i, j+0.5}^{n}-G_{i, j-0.5}^{n}}{\Delta y}\right)
$$

A estratégia consiste em escrever esta equação para todos os pontos do domínio computacional, gerando um sistema de equações lineares $\overrightarrow{A p}=\vec{b}$, onde $\vec{b}$ e $\vec{p}$ guardam os valores $b(i, j)$ e da pressão, respectivamente, em cada célula.

Solucionando numericamente este sistema, é possível substituir $p^{n+1}$ em (5) e (6), a fim de atualizar os valores de $u_{i+0.5, j}^{n+1}$ e $v_{i, j+0.5}^{n+1}$, avançando todas as variáveis do escoamento no tempo.

São necessários, ainda, alguns ajustes nos valores das variáveis com base no valor do divergente em cada volume de controle, a fim de determinar um campo de velocidades que satisfaça, simultaneamente, as equações de conservação da massa e de conservação do momento, dentro dos limites dos erros das discretizações e arredondamentos nos cálculos. Este procedimento é adequadamente descrito em [3]. 


\section{Abordagem computacional}

\subsection{Autonomous Leaves Graph (ALG)}

O ALG é uma estrutura de dados baseada em grafos proposta em [2] como ferramenta para manipulação adaptativa de malhas não uniformes e auxílio na resolução numérica de EDP's. Em cada passo da execução do algoritmo, uma determinada região da malha sofre um refinamento ou desrefinamento, de acordo com o objetivo do estudo ou comportamento da solução do modelo em questão. A descrição e as ilustrações utilizadas nesta seção foram extraídas de [1].

Para descrever o procedimento usado pelo ALG, considere inicialmente um domínio bidimensional formado por um quadrado unitário, como ilustrado na Figura 2. Dividindo este quadrado em quatro quadrados iguais obtêm-se quatro células, cujos centros são assinalados com um vértice preto. É possível esboçar este esquema por meio de um grafo. Por meio de arestas, cada vértice preto apontará para quatro direções, denotadas por Norte $(N)$, Sul $(S)$, Leste $(L)$ e Oeste $(\mathrm{O})$, cada qual apontando para um vértice branco, inserido com o propósito de conectar níveis de refinamento diferentes.
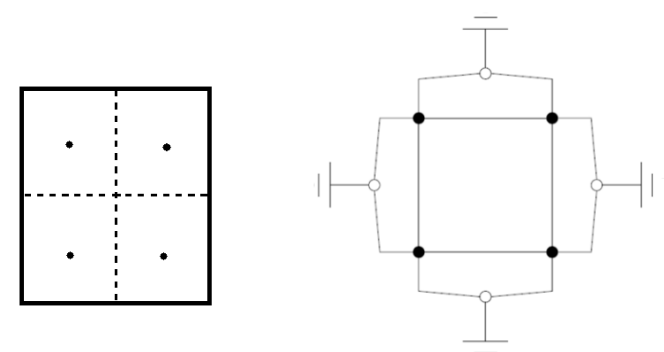

Figura 2: Subdivisão de células e construção do grafo.

Subdividindo um dos quadrados, será substituído por um novo cacho com quatro vértices, todos indexados com o número 2, indicando o nível de refinamento destes vértices, como ilustrado na Figura 3 abaixo. Este procedimento é, então, repetido sobre determinadas células ou regiões da malha, de acordo com a configuração de refinamento que se deseja obter.
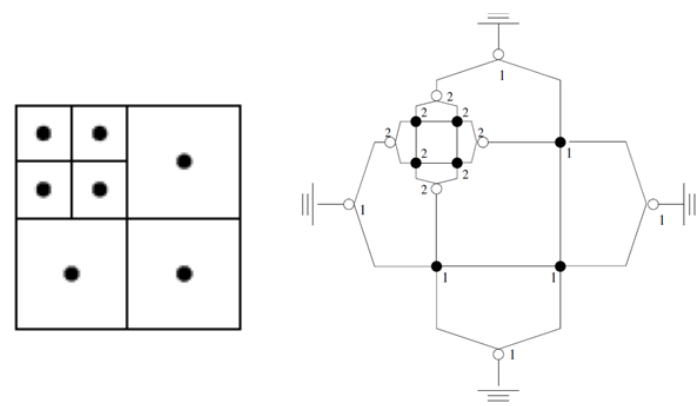

Figura 3: Refinamento nível 2
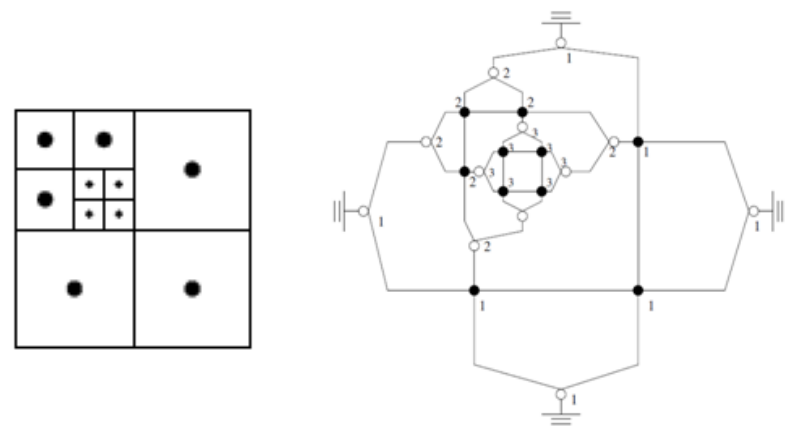

Figura 4: Refinamento de nível 3 


\subsection{Domínio}

O domínio de observação do problema é, portanto, um quadrado unitário, representando a superfície do escoamento. Foi utilizada a configuração de um canal, com fronteiras acima (Norte) e abaixo (Sul) da região simulando paredes de contenção para o escoamento, além das fronteiras de entrada e saída do fluxo, que segue no sentido Oeste-Leste. Uma estrutura aproximadamente circular foi inserida no centro do domínio para simular a presença de um tronco, servindo de obstáculo ao escoamento. Esquematicamente, tem-se:

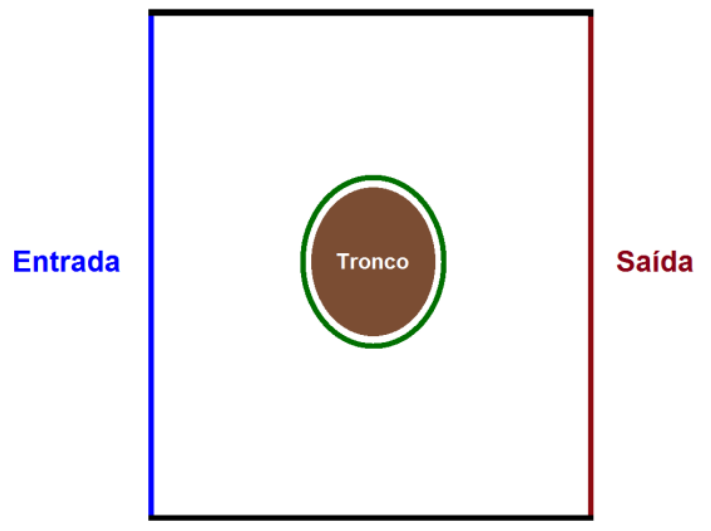

Figura 5: Domínio de observação do problema.

Computacionalmente, toda a região ilustrada na Figura 5 é representada por uma malha construída pelo ALG. As células da malha que correspondem ao círculo verde recebem as mesmas condições de contorno estabelecidas nas fronteiras Norte e Sul do domínio, a fim de que configure efetivamente um obstáculo. Já as células que estão dentro desta borda são inativas, indicando a impossibilidade de serem usadas nos cálculos.

As condições de contorno consideradas são:

- Fronteira Oeste (Entrada): $u=10, v=0, p=1$

- Fronteira Leste (Saída): $u_{x}=0, v_{y}=0, p_{x}=p_{y}=0$

- Fronteiras Norte, Sul e tronco: $u=0, v=0, p=0$

\section{Resultados}

Para o problema estudado, o ALG executa a sequência de operações abaixo:

Entrada: Condições iniciais e de contorno;

Refinamento inicial;

Para $t<t_{\text {final }}$ :

$$
\begin{gathered}
\text { Enquanto (solução não converge) } \\
\text { Calcula pressão; } \\
\text { Calcula velocidades; } \\
\text { Ajusta pressão; } \\
\text { Ajusta velocidades. } \\
\text { Fim enquanto; }
\end{gathered}
$$

Fim para.

Na Figura 6 abaixo tem-se a representação do campo de direções do vetor velocidade em cada célula da malha. Esta informação é obtida a partir da média entre a velocidade na direção $x$ e a velocidade na direção $y$. Vale ressaltar que este resultado não traduz a intensidade das 
velocidades, mas, apenas, a direção. Os vetores foram formatados de modo que tivessem origem no centro de cada célula e ficassem contidos dentro das mesmas.

Na Figura 6 (a), para delimitar a borda do tronco foram marcadas em verde as células que estão a um raio de 0.1 da célula $(0.5,0.5)$, isto é, do centro do quadrado que demarca o domínio. Já na Figura 6 (b), os troncos têm raio 0.07 e estão centrados em $(0.5,0.25)$ e $(0.5,0.75)$. As células nestas bordas são os troncos propriamente ditos. Os círculos internos são apenas ilustrativos e estão sobrepostos a células inativas.

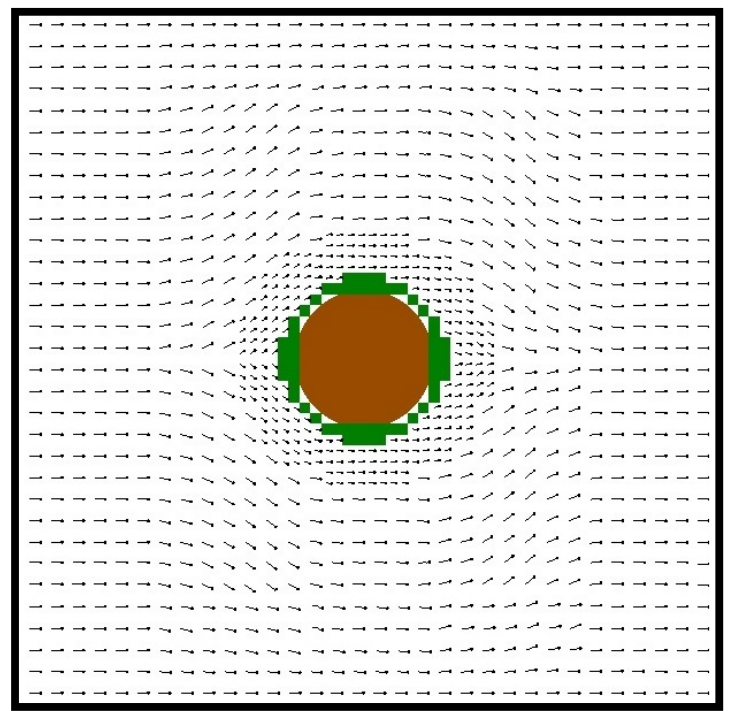

(a)

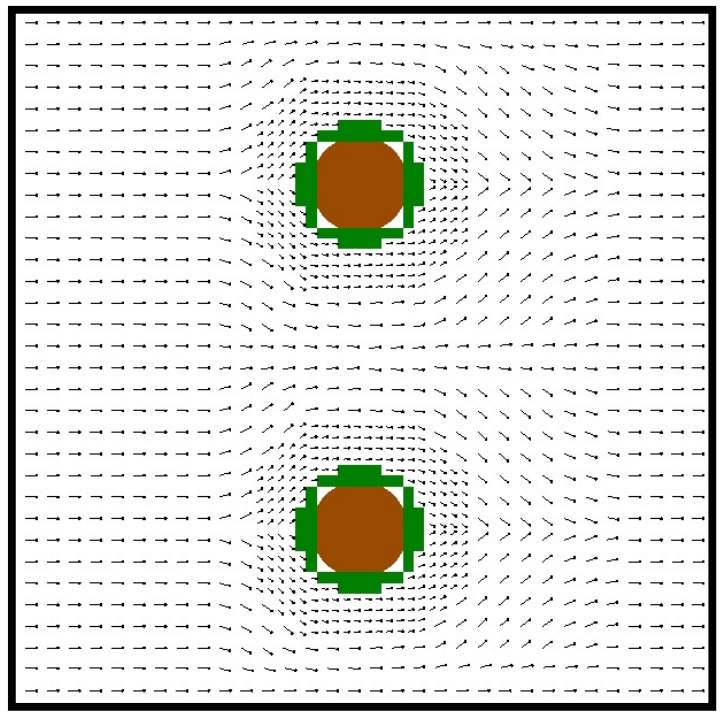

(b)

Figura 6: Simulação com um tronco (a) e dois troncos (b) como obstáculos ao escoamento.

O critério de convergência utilizado é referente a diferenças entre os valores das variáveis, em passos de tempo consecutivos, que devem ser menores que um erro previamente estabelecido.

A estimativa dos valores da pressão no escoamento, bem como a representação das intensidades das variáveis, serão alvo de trabalhos futuros.

\section{Referências}

[1] BURGARELLI, D.; "Modelagem computacional e simulação numérica adaptativa de equações diferenciais parciais evolutivas aplicadas a um problema termoacústico". Tese de doutorado, PUC-Rio, Rio de Janeiro, Brasil, 1998.

[2] BURGARELli, D., KISCHINHEVSKY, M., BIEZUNER, R. J.; “A new adaptive mesh renement strategy for numerically solving evolutionary PDEs". Journal of Computational and Applied Mathematics, 196, páginas 115-131, 2006.

[3] FORTUNA, A. O.; "Técnicas Computacionais para Dinâmica de Fluidos, Conceitos Básicos e Aplicações”, Edusp, 2000.

[4] KRITZ, M. V.; DIAS, C. M.; SILVA, J. M.; "Modelos e Sustentabilidade nas Paisagens Alagáveis Amazônicas"; Notas em Matemática Aplicada, V.34, SBMAC, 2008.

[5] LEAL, T. F.; "Simulação numérica do escoamento de água em áreas alagáveis da floresta amazônica com a utilização da estrutura de dados Autonomous Leaves Graph", Dissertação de Mestrado em Ciências Computacionais, Instituto de Matemática e Estatística, Universidade do Estado do Rio de Janeiro, 2013.

[6] MOURA, C. A.; BURGARELLI, D.; "Tópicos de Análise Funcional na Computação Científica"; Notas em Matemática Aplicada, V.45, SBMAC, 2010. 